\title{
Essai D'evaluation Des Gisements De Dechets Organiques Animaux D'abattage Dans La Perspective D'une Planification De Leur Gestion Durable Dans Le Grand Nokoue Au Sud-Benin
}

\section{Moussa Wari Aboubakar}

Laboratoire Pierre PAGNEY: Climat, Eau, Ecosystèmes et Développement Centre de Valorisation des Déchets en Energie et Agriculture (ValDERA)

\section{Gratien Boni}

Centre Interfacultaire de Formation et de Recherche en Environnement pour le Développement Durable (CIFRED)

\section{Placide Cledjo}

Laboratoire Pierre PAGNEY: Climat, Eau, Ecosystèmes et Développement Centre de Valorisation des Déchets en Energie et Agriculture (ValDERA), Centre Interfacultaire de Formation et de Recherche en Environnement pour le Développement Durable (CIFRED)

Doi: 10.19044/esj.2018.v14n30p477 URL:http://dx.doi.org/10.19044/esj.2018.v14n30p477

\section{Abstract}

The slaughterhouse of Cotonou and the surfaces of slaughtering of Porto-Novo, Abomey-Calavi, Sèmè-Kpodji and Ouidah constitute sources of animal solid matter flow in Large Nokoué. Within the framework of this research, it is refers to the contents of paunches considered for their potential of valorization in the production of biogas and the compost. Then, the quantitative evaluation their layers is a contribution to the efficient use of those as resources or raw materials, for a planning of future management. The methodological approach used is centered on the document retrieval, the datagathering by measurements or weighing of the roughs weight of contents of paunch of the animal species using balance of brand CAMRY as well as recovery of the statistics of slaughtering in the structures in charge of the breeding and the services of inspection of the activities of slaughtering units. The data obtained were treated then the analyzed results. The sample size selected is of 50 individuals of species cattle and 50 individuals of species of caprine sheep. The data analysis was analyzed, inter alia, thanks to software SPSS version 17.0 was used for the statistical analyses. It comes out from this research that each shot down cattle generate $48.5612 \mathrm{~kg}$ of waste (contained digestive). It is advisable to recall that the majority of the cattle taken as 
sample is race zebu and bull-fighting. As for the sheep and goats, each animal generates a quantity of approximately $4.0364 \mathrm{~kg}$ of waste. With this average of $48.5612 \mathrm{~kg}$ of contents of bovine paunch, and $4.0364 \mathrm{~kg}$ for the sheep and goats, the layer of contents the digestive ones was estimated at 1060 tons for the year 2011, and at 1298 tons for the year 2015 at the level of Large Nokoué. If this trend is maintained, this layer could border 2011 tons by 2030. The evaluation of the layers of contents of paunch produces by the activities of demolition the sector of the study regarded as largest parts of the fractions which may undergo beneficiation of the livestock wastes takes part in the installation of strategies of perpetuation of the actions of treatment and valorization within the framework of a sector.

Keywords: Slaughtering units, layers, contents of paunch, projection

\section{Resume}

L'abattoir de Cotonou et les aires d'abattage de Porto-Novo, AbomeyCalavi, Sèmè-Kpodji et Ouidah constituent des sources de flux de matières solides animales dans le Grand Nokoué. Dans le cadre de cette recherche, il s'agit des contenus de panses considérés pour leur potentiel de valorisation dans la production du biogaz et du compost. Alors, l'évaluation quantitative de leurs gisements est une contribution à l'utilisation efficiente de ceux-ci en tant que ressources ou matières premières, en vue d'une planification de la gestion future. La démarche méthodologique utilisée est axée sur la collecte des données par les mesures ou pesée des masses brutes de contenus de panse des espèces animales à l'aide de balance de marque CAMRY ainsi que la récupération des statistiques d'abattage dans les structures en charge de l'élevage et les services d'inspection des activités d'abattages. Les données obtenues ont été traitées puis les résultats analysés. La taille de l'échantillon retenu est de 50 individus d'espèce bovin et 50 individus d'espèce d'ovins caprins. L'analyse des données a été faite, entre autres, à l'aide du logiciel SPSS version 17.0 qui a été utilisé pour les analyses statistiques. Il ressort de cette recherche que chaque bovin abattu génère $48,5612 \mathrm{~kg}$ de déchets (contenus digestif). Il convient de rappeler que la majorité des bovins pris comme échantillon est de la race zébu et taurin, reformuler. Quant aux ovins et caprins, chaque animal génère une quantité de d'environ 4,0364. $\mathrm{kg}$ de déchets. Avec cette moyenne de 48,5612 $\mathrm{kg}$ de contenus de panse bovine, et 4,0364 kg pour les ovins et caprins, le gisement de de contenus de digestifs a été estimé à 1060 tonnes pour l'année 2011, et à 1298 tonnes pour l'année 2015 à l'échelle du Grand Nokoué. Si cette tendance est maintenue, ce gisement pourrait avoisiner 2011 tonnes d'ici 2030 à, partir de quelle methode de prevision avez-vous obtenu ce resultat. L'évaluation des gisements de contenus de panse produit par les activités d'abattage dans le secteur d'étude 
considérés comme la plus grande partie des fractions valorisables des déchets animaux. Cette évaluation participe à la mise en place de stratégies de pérennisation des actions de traitement et de valorisation dans le cadre d'une filière à travers la connaissance des quantités de déchets disponibles.

Mots clés : Unités d'abattage, gisements, contenus de panse, projection

\section{Introduction}

L'accroissement de la population urbaine dans les villes des pays du sud comme ceux du nord est l'une des principales causes de l'augmentation de la production des déchets (Tanawa et al., 1997 ; Onibokun, 2001 ; Citeretse 2008 ; Ngambi, 2015) les references doivent suivre un ordre chronologique. Par ailleurs, le phénomène de mondialisation ne limitant plus les échanges entre sociétés a permis aux populations de s'arrimer aux modes de consommations des sociétés modernes. Ceci a non seulement contribué à diversifier diversifié la qualité des déchets mais est à la source de l'augmentation de la production des ordures ménagères dans les villes africaines (Ngambi, 2015). Kapepula (1996) montre que la quantité des déchets produits par les grandes villes africaines, en l'occurrence les capitales, n'a cessé d'augmenter de 1960 à nos jours et probablement d'atteindre pour l'an $2015,100 \%$ à plus de $300 \%$ de la production initiale. Le rapport entre la production et la collecte des déchets solides ménagers (DSM) dans les villes d'Afrique reste encore faible et ceci constitue un épineux problème pour la qualité de l'environnement et la sécurité sanitaire des habitants.

La connaissance des gisements de déchets, en termes quantitatif et qualitatif, est d'une importance fondamentale dans la mise en place d'une politique de gestion. Ces informations sur les gisements sont particulièrement déterminantes à 2 niveaux de la planification de la gestion comme le soulignent Tchobanoglous et al. (1993): il s'agit, premièrement de l'évaluation des potentiels de diversion ou de réduction, de recyclage et valorisation de certaines catégories de déchets ; et deuxièmement du choix des matériels et équipements, l'aménagement des sites de stockage et d'élimination, la définition des circuits de collecte, l'allocation et la répartition des moyens. De nombreux travaux ont été réalisés aux fins d'évaluer les gisements de déchets organiques en particulier citez-en quelques-uns, d'une part comme générateurs potentiels de risques pour l'environnement et la santé publique et/ou d'autre part comme ressources potentiellement valorisables, en fonction de leurs propriétés bio-physico-chimiques (Lacour, 2012). En effet, selon Lacour (2012), l'utilisation de résidus de cultures dans les zones rurales et périurbaines, a été l'objet de recherche et de développement soutenus, dans différents endroits à travers le monde, dans les années 1970. Ce nouveau paradigme a débuté à la suite des publications de Lampila (1963) et de Wilson 
et Pigdon (1964) et le développement de technologies d'amélioration des propriétés digestives de la paille de céréales dans l'alimentation animale, au niveau de l'Europe et de l'Amérique du Nord, avant de s'étendre au niveau des pays tropicaux en développement, en particulier l'Inde, le Bangladesh et d'autres pays de l'Asie et de l'Afrique, dans un mouvement qui pourrait être qualifié comme une « révolution des résidus » à partir de 1980 (Owen et Jayasuriya, 1989). Parallèlement, dans la même période, les incidences des fractions organiques de déchets urbains sur la définition et les opérations des systèmes de collecte et sur les travaux d'aménagement et de gestion des sites de traitement, ont bénéficié d'une attention accrue, en raison de la décomposition rapide de celles-ci, notamment dans les régions à climat chaud (Tchobanoglous et $a l .$, 1993). Ces processus ont conduit à la généralisation des pratiques d'estimation ou d'inventaire des gisements de déchets organiques, à la fois dans les pays industrialisés et dans les PED, soit à des fins de planification des systèmes de gestion, soit pour en évaluer les potentiels de valorisation. Dans le cas du Grand Nokoué, aucune étude systémique de quantification et de caractérisation des gisements de fractions organiques des déchets animaux, n'a été recensée à ce jour. Dès lors, un travail de recherche dans ce cadre s'avère pertinent dans les 2 cas de figures alternatifs les plus envisageables. D'abord, dans le cas où les déchets organiques sont assimilés à des sources de pollution et nuisance, la connaissance de leurs gisements participe d'une évaluation des risques pour l'intégrité des écosystèmes et pour la santé des populations exposées à des conditions d'extrême précarité. Ensuite, dans l'autre cas où ces déchets et résidus organiques sont considérés pour leur potentiel de valorisation matière et/ou énergie, même sommaire. L'évaluation quantitative et qualitative de leurs gisements peut contribuer à une utilisation efficiente de ceux-ci en tant que ressources, particulièrement dans ces contextes de déficit d'accès aux infrastructures de base et aux moyens de production. La présente recherche s'inscrit principalement en droite ligne du second cas de figure. Elle propose une quantification en termes de masse des gisements de déchets organiques animaux (contenus de panse), à partir des estimations des abattages rapportés aux unités aux abattoirs et assimilés du Grand Nokoué.

\section{Cadre d'Etude}

L'étude s'est déroulée au Sud-Bénin dans le Grand Nokoué qui est un territoire constitué de cinq communes dont deux du département de l'Atlantique (Abomey-Calavi, Ouidah); deux du département de l'Ouémé (Porto-Novo, Sèmè-Kpodji) et Cotonou. Le Grand Nokoué couvre une superficie de $1252 \mathrm{~km}^{2}$ et est située entre $6^{\circ} 15^{\text {ee }}$ et $6^{\circ} 45^{\prime \prime}$ de latitude nord et $2^{\circ} 0^{\prime \prime}$ et $2^{\circ} 45^{\prime \prime}$ de longitude est (figure 1 ). 


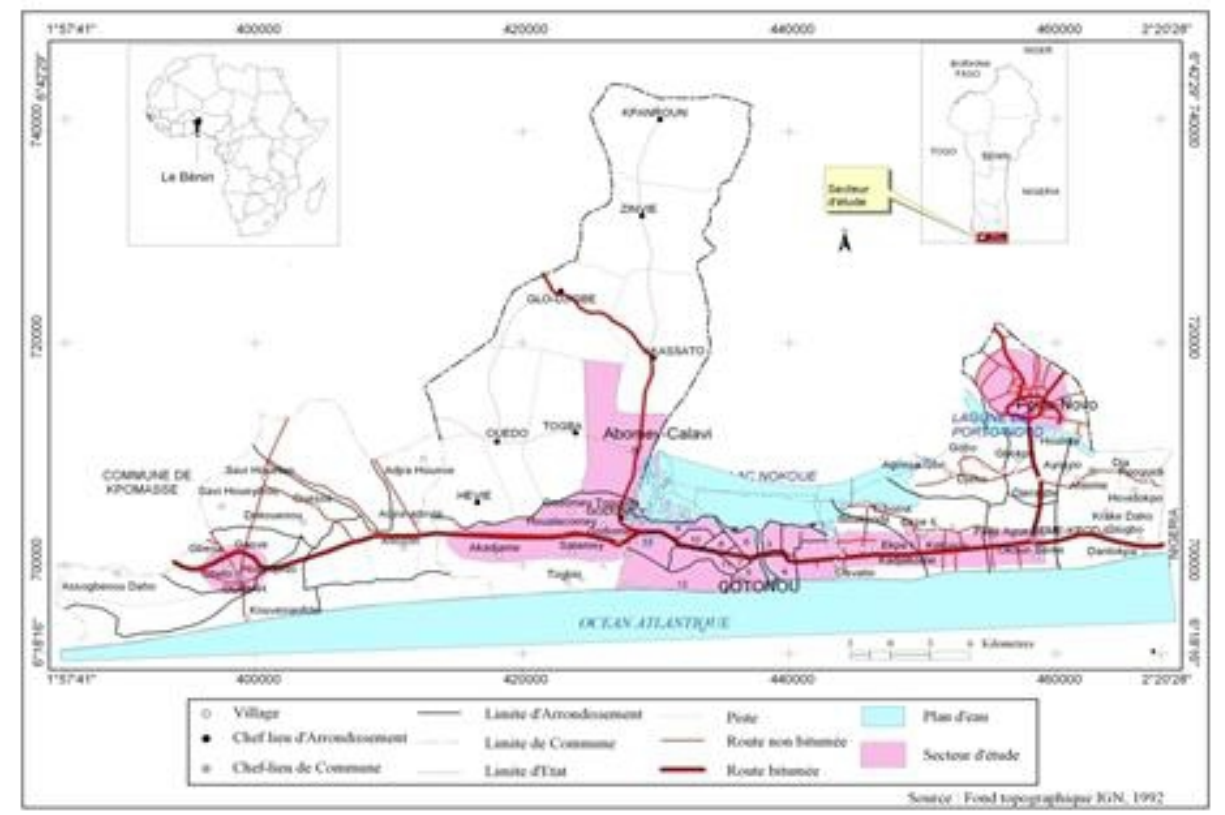

Figure 1 : Situation géographique du Grand Nokoué

L'analyse de la figure1 montre les limites dans lesquelles s'est menée la présente étude et qui porte uniquement sur les agglomérations urbaines du Grand Nokoué à savoir Porto-Novo, Semè-Podji, Cotonou, Abomey-CalaviGodomey et Ouidah.

\section{Outils et Methodes de Collecte et de Traitement des Donnees}

Il s'agit d'une étude quantitative, prospective et analytique. Dans le but d'atteindre l'objectif visé par cette recherche, il a été adopté une méthodologie qui s'articule autour de la collecte de données de terrain, le traitement et l'analyse des résultats. Cette méthode se base sur les statistiques d'abattage et surtout la prise mesures in situ des quantités de déchets solides organiques produit par une espèce animale considérée. Il faut dire, jusqu'ici, les données de ce type ne sont pas encore disponible dans le secteur d'étude.

\section{Outils de collecte et de traitement des données}

Les outils utilisés pour la collecte des données sont entre autres : le questionnaire, le guide d'entretien, la grille d'observation, le GPS pour la prise des coordonnées des unités d'abattage, l'appareil photo numérique pour la prise de vues, un micro-ordinateur pour la saisie et le traitement des données, des balances mécaniques CAMRY pour la pesée des déchets cible. 


\section{Collecte de données sur le terrain}

La présente recherche s'est fixée comme objectif de procéder à une quantification en termes de masse des gisements de déchets organiques, à partir des estimations du nombre de têtes d'ovins/caprins et de bovins abattu au niveau des sites d'abattage identifiés. Mais, il ne s'agit point de prétendre à une évaluation très précise des diverses catégories de déchets organiques générés durant la période de référence (2011 à 2015) au niveau des unités d'abattage cibles. Ainsi, pour n'avoir tenu compte que de certains gisements bien spécifiques de déchets et résidus organiques, ces résultats ont une valeur d'indication par défaut, c'est-à-dire offrant au moins la certitude que les quantités de déchets organiques disponibles sont supérieures ou égales aux estimations de l'étude. De plus, les ordres de grandeur inférieurs généralement retenus, dans le cas des estimations ouvertes, contribuent à une sousestimation des résultats, selon une hypothèse pessimiste (Lacour, 2012). Ainsi, les contenus digestifs et/ou matières stercoraires sont les catégories de déchets qui sont considérés. Car, ils constituent la majeure partie des déchets solides animaux des unités d'abattage.

Le nombre d'animaux abattus au niveau des différents sites d'abattage pour les cinq années (2011 à 2015) a été obtenu au niveau de l'abattoir de Cotonou et des services de contrôle vétérinaires des ex CARDER AtlantiqueLittoral et Ouémé-Plateau. Ensuite la moyenne de la dynamique d'abattage a été établie par comparaison des données des années en présence. En effet, la moyenne consiste en la valeur résultante de la division de la différence entre les statistiques du nombre d'abattage des années 2011 et 2015 par le nombre d'année qui est 5. La moyenne est qualifiée de progressive lorsque la moyenne est positive, et régressive lorsqu'elle est négative. Cette valeur moyenne obtenue par site d'abattage a permis d'estimer le nombre d'animaux (Bovins et ovins/caprins) abattus sur chaque site jusqu'en 2030 selon l'hypothèse basée sur les extensions linéaires du nombre d'animaux abattus observé sur chaque site entre 2011 et 2015 , en considérant :

- l'environnement institutionnel inchangé,

- les déterminants sociaux inchangés,

- les bases humaines et matérielles du développement non favorable,

Pour déterminer la quantité de déchets animaux (contenus digestifs ( $\boldsymbol{W}_{\text {aiy }}$ ) produite par la quantité d'espèce animale abattue (ai), au cours de l'année de référence $(\boldsymbol{y})$, la production moyenne par animale de déchets animaux par individu $\left(\boldsymbol{W}_{\text {aid }}\right)$ est multipliée par l'effectif d'abattage $\left(\boldsymbol{A}_{\text {iy }}\right)$ recensée pour la période de référence. La somme des gisements de déchets des espèces retenues dans le cadre de l'évaluation, est une sous-estimation de la quantité totale de déchets animaux produite par l'ensemble des unités d'abattage, au cours de l'année y $\left(\boldsymbol{W}_{\text {aty }}\right)$. Ces expressions sont traduites par les relations 1 et 2 : 


$$
\begin{gathered}
\boldsymbol{W}_{\text {aty }}\left(\text { tonnes } / \text { an } \geq \sum_{i=1}^{n} \boldsymbol{W}_{\text {aid }}(\text { tonnes } / \text { an }(3)(1)\right. \\
\boldsymbol{W}_{\text {aiy }}(\text { tonnes } / \text { an })=\boldsymbol{W}_{\text {aid }}(\text { tonnes } / \text { animal }) \times \boldsymbol{W}_{\text {aiy }}\left(\text { nombre de } \frac{\text { tête }}{\text { an }}\right)
\end{gathered}
$$

Le nombre de bovins et ovins/caprins ont été obtenus à partir des statistiques de l'abattoir de Cotonou et celles des services de contrôle vétérinaires des CARDER Atlantique-Littoral et Ouémé-Plateau considérées pour une période de cinq ans de référence (y1 à $\mathrm{y}_{5}$ ) 2011 à 2015, relatives aux quantités d'abattage des espèces bovines (fi) et espèces ovins/caprins (ai), multipliés par des indices résiduels respectifs estimés pour les différentes unités d'abattage (ci) ou par des indices de production spécifique de contenus digestifs animaux des espèces considérés ( Adapté d'Owen et Jayasuriya, 1989, rapporté par Lacour ( 2012). Le mode d'abattage, l'espèce, le nombre de têtes, de même que le poids des individus par espèce ont été pris en compte, ce qui a finalement permis de quantifier le total de déchets animaux produites par une unité d'abattage chaque année et pour toutes les années de la période prise comme référence.

La méthodologie proposée prend seulement en compte les productions de déchets animaux des bovins, des ovins et des caprins. Il s'agit ici des déchets stercoraires ou contenus digestifs des bovins, des ovins et des caprins qui constituent la majeure partie des déchets rejetés par jour. Ainsi, pour évaluer la quantité de ces déchets produits, une campagne de pesés des produits de chaque espèce a été procédée pendant 50 jours. Il faut préciser ici que les 50 jours ont été subdivisés en deux sous-périodes dont 25 jours en période de pluie (juin 2017) et 25 jours en période de sécheresse (février 2017). Le processus a été conduit de telle manière que chaque individu animal de l'échantillon est pesé avant son abattage, et ceci relever son poids vif. Juste après son abattage, suit immédiatement la pesée de son contenu digestif. Il faut préciser que, chaque jour, il est procédé à un choix aléatoire parmi les bêtes en attente d'être abattu, dont une espèce bovine et une espèce d'ovin/caprin, et ces opérations ont été faites à $80 \%$ à l'abattoir de Cotonou, compte tenu de l'importance des effectifs d'animaux abattus. Les $20 \%$ restants ont été répartis dans les aires d'abattage cibles, tenant compte de la proportion d'abattage par rapport à l'abattoir de Cotonou. Après le choix, l'espèce numérotée dans un ordre d'abattage est suivie jusqu'à l'étape d'éviscération, où les apprentis récupèrent les panses pour les conduire sur le lieu de vidange où le pesage se fait immédiatement avec leur contenu puis ensuite le contenu seul afin pouvoir procéder à des comparaisons. Le poids du contenu de chaque espèce ainsi obtenu est ensuite relevé et inséré dans la base de données Excel crée à cet effet. A l'issu de ces opérations, les données ont été normalisées et consolidées, puis, insérées dans un modèle. Afin de la 
détermination de la quantité de déchet par année, le poids moyen des déchets par espèce a été obtenu par les formules suivantes

1) Soit $\mathrm{P}_{\mathrm{bm}}$ le poids moyen de déchets de bovin On a $: \mathrm{P}_{\mathrm{bm}}=\frac{P_{b}}{50}$

Avec $\mathrm{Pb}=$ Poids total des déchets obtenus pour les 50 têtes choisis

2) Soit $P_{\text {om }}$ le poids moyen de déchets d'ovin/ caprin, $P_{\text {om }}=\frac{P_{O}}{50}$

Avec $\mathrm{Po}=$ poids total des déchets d'ovin/caprin et $\mathrm{Pb}=$ Poids total des déchets de bovins obtenus pour les 50 têtes choisis pour chaque espèce.

\section{Traitement des données et analyse des résultats}

Après le dépouillement des fiches d'enquête et la codification des données, une base de données a été constituée avec les logiciels Excel et SPSS version 17.0. Ensuite, quelques outils de la statistique descriptive ont été mis à contribution à savoir : la moyenne arithmétique, le pourcentage, la fréquence (relative et absolue) et les illustrations graphiques. Une analyse de la variance non paramétrique sur mesures répétées à un facteur a été nécessaire pour l'analyse des données liées à la quantification des déchets.

\section{Resultas et Discussions}

\section{Estimation des quantités de matières organiques des déchets solides animaux}

Le présent exercice propose une quantification en termes de masse des gisements de déchets organiques (contenus de penses ou contenus digestifs), étant donné que la présente s'inscrit dans le cadre de la valorisation de ce type de déchet en énergie et compost. Cette évaluation est faite à partir des estimations des abattages spécifiques des bovins, ovins et caprins rapportées aux unités d'abattage ci-dessus citées. Il ne s'agit pas forcément d'une évaluation exhaustive des diverses catégories de déchets organiques générés, durant leurs périodes et lieux de production respectifs, par des activités d'abattage. Ainsi, pour n'avoir tenu compte que de certains gisements bien spécifiques de déchets et résidus organiques d'abattage séparés à la source, fut-ils les fractions solides les plus importantes en termes de masse, ces résultats ont une valeur d'indication par défaut, c'est-à-dire offrant au moins la certitude que les quantités de déchets organiques disponibles sont supérieures ou égales aux estimations de l'étude. Donc, il serait juste de penser que l'estimation des quantités de déchets animaux produits est exhaustive, en raison des variations saisonnières qui se produisent d'une année à l'autre, mais aussi par le fait qu'une quantité importante de ces résidus est issue des abattages en dehors des sites contrôlés. En conséquence, les chiffres des quantités de déchets animaux sont estimés à partir des effectifs de bêtes abattues et contrôlées par les unités d'abattages. 
Evolution des effectifs d'abattage dans les unités d'abattage ciblées de 2011 à 2015

Le nombre de bovins et ovins/caprins ont été obtenus à partir des statistiques de l'abattoir de Cotonou et celles des services de contrôle vétérinaires des ex CARDER Atlantique-Littoral et Ouémé-Plateau considérées pour une période de cinq ans de référence 2011 à 2015, relatives aux quantités d'abattage des espèces bovines (fi) et espèces ovins/caprins (ai). Ces données ont été complétées par les celles obtenues sur le terrain. Le tableau I présente les statistiques d'abattage des différentes espèces prises en comptes dans le cadre de cette étude et par unité d'abattage de 2011 à 2015 et qui permis de corréler les effectifs abattus avec les quantités de déchets qui en ont découlées. 
Tableau I : Statistiques d'abattage des différentes espèces prises en comptes par unité d'abattage de 2011 à 2015

\begin{tabular}{|c|c|c|c|c|c|c|c|c|c|c|c|}
\hline \multirow[t]{2}{*}{$\mathbf{N}^{\circ}$} & \multirow[t]{3}{*}{ IDENTIFIANT } & & & & & \multicolumn{6}{|c|}{ ANNEES } \\
\hline & & \multicolumn{2}{|r|}{2011} & \multicolumn{2}{|r|}{2012} & \multicolumn{2}{|r|}{2013} & \multicolumn{2}{|c|}{2014} & \multicolumn{2}{|r|}{2015} \\
\hline & & Bovins & Ovin/caprin & Bovins & Ovin/caprin & Bovins & Ovin/caprin & Bovins & Ovin/caprin & Bovins & Ovin/caprin \\
\hline 1 & Abattoir de Cotonou & 13700 & 54174 & 15679 & 101629 & 15068 & 77850 & 15633 & 81908 & 15666 & 81030 \\
\hline 2 & Aire d'abattage de Sèmè-Kpodji & - & 4098 & - & 7491 & - & 6247 & - & 4353 & - & 6641 \\
\hline 3 & Aire d'abattage de Porto-Novo & 1329 & 4762 & 1222 & 3418 & 1170 & 4008 & 1181 & 9889 & 1244 & 6663 \\
\hline 4 & Aire d'abattage d'Abarame & 330 & - & 329 & - & 472 & - & 472 & - & 474 & - \\
\hline 5 & ¿্ট̈ · Aire d'abattage de Kpota & 440 & 3915 & 438 & 3916 & 360 & 3951 & 630 & 2714 & 631 & 4040 \\
\hline 6 & : Aire d'abattage la fontaine & 110 & - & 109 & - & 157 & - & 157 & - & 158 & - \\
\hline 7 & . Aire d'abattage d'Agbanou & 92 & 342 & 89 & 371 & 91 & 371 & 93 & 377 & 97 & 368 \\
\hline 8 & Aire d'abattage de Gomey & 88 & 365 & 79 & 371 & 81 & 367 & 73 & 377 & 87 & 368 \\
\hline 9 & $\begin{array}{l}\text { 'Aire d'abattage de Zomaï } \\
\text { TOTAUX }\end{array}$ & $\begin{array}{c}102 \\
\mathbf{1 6 0 8 9}\end{array}$ & $\begin{array}{c}342 \\
\mathbf{7 0 0 0 9}\end{array}$ & $\begin{array}{c}91 \\
\mathbf{1 7 9 4 5}\end{array}$ & $\begin{array}{c}371 \\
\mathbf{1 1 9 5 7 9}\end{array}$ & $\begin{array}{c}109 \\
\mathbf{1 7 3 9 9}\end{array}$ & $\begin{array}{c}357 \\
\mathbf{9 5 1 6 4}\end{array}$ & $\begin{array}{c}104 \\
\mathbf{1 8 2 3 9}\end{array}$ & $\begin{array}{c}377 \\
\mathbf{1 0 2 0 0 9}\end{array}$ & $\begin{array}{c}112 \\
\mathbf{1 8 3 5 7}\end{array}$ & $\begin{array}{r}368 \\
101493\end{array}$ \\
\hline
\end{tabular}


Il ressort de l'analyse du tableau I, les effectifs d'espèces bovines et ovines/caprin ont variées selon es unités d'abattage et ont connu une tendance progressive de de 2011 à 2015. D'après les données du tableau, il se dégage clairement que les capacités d'abattage de l'abattoir de Cotonou sont de loin nettement supérieures à celles de toutes les aires d'abattage. On peut noter que les quantités d'abattage annuelles à l'abattoir de Cotonou sont de 13700 en 2011 à 15666 têtes d'espèces bovines en 2015. Pour la même période, on est passé de 54174 têtes de ovins/ caprins en 2011 à 81030 têtes en 2015. Soit respectivement des taux d'accroissement respectifs de $12,54 \%$ pour ce qui est des bovins, et 33,14\% en ce qui concerne les ovins caprin, le tout en 5 ans.

A l'aire d'abattage de Porto-Novo, les données du tableau I montrent que les quantités d'abattage annuelles sont de 1329 en 2011 à 1244 têtes d'espèces bovines en 2015. Pour la même période, ce nombre passé de 4762 têtes de ovins/ caprins en 2011 à 6633 têtes en 2015, soit un taux de progression de $28,2 \%$. Contrairement aux effectifs d'abattage des ovins / caprins qui ont connu une croissance de 2011 à 2015, les effectifs des bovins ont connu une tendance baissière, soit de régression de $-6,83 \%$.

A l'aire d'abattage de Sèmè-Kpodji où il n'est abattu que les caprin et ovin, les données du tableau montrent que les quantités d'abattage annuelles sont de 4098 têtes de ovins/ caprins en 2011 à 6641 têtes en 2015, soit un taux de progression de $38,29 \%$.

Pour ce qui concerne les trois aires d'abattage de la commune de d'Abomey-Calavi, on observe que les effectifs d'abattage annuels sont passés de 330 têtes de Bovins en 2011 à 474 en 2015 à Abaramè ; ils sont passés de 110 têtes de Bovins en 2011 à 158 en 2015 à la Fontaine. Pour ces deux aires, seuls les bovins sont abattus ou recensés au moment où l'étude se déroulait. Par contre, dans l'aire de Kpota, il est abattu aussi bien les bovins que les ovins et caprins. Ainsi, dans l'aire de Kpota, les données du tableau montrent que les quantités d'abattage annuelles sont de 440 en 2011 à 631 têtes d'espèces bovines en 2015. Pour la même période, on est passé de 3915 têtes de ovins/ caprins en 2011 à 4040 têtes en 2015.

Dans la commune de Ouidah, où dénombre trois aires d'abattage aussi, les données du tableau montrent que les effectifs d'abattage annuels sont de 92, 88 et 102 en 2011 à 97, 87 et 112 têtes d'espèces bovines en 2015, respectivement dans les aires d'abattage d'Agbanou, de Gomey et Zomaï. Pour ce en ce qui concerne les ovins /caprins, elles sont respectivement passées de 342, 365 et 342 en 2011 à 368, 368 et 368 en 2015, respectivement dans les aires d'abattage d'Agbanou, de Gomey et Zomaï.

Donc au total, pour l'ensemble des aires d'abattage, les quantités d'abattage des bovins sont de 16089 en 2011, de 17945 en 2012, de 17399 en 2013, de 18239 en 2014 et de 18357 en 2015. Pour l'ensemble des aires d'abattage, les quantités d'abattage des ovins et caprins sont de 70009 en 2011, 
de 119579 en 2012, de 95164 en 2013, de 102009 en 2014 et de 101493 en 2015. Sur la base de ces effectifs annuels d'abattage par unité d'abattage, on a pu effectuer une projection jusqu'en 2030, pour voir quelles seraient les capacités d'abattage.

\section{Résultats des projections des effectifs d'abattage d'ici 2030}

Il s'agit bien des effectifs des espèces considérées pour le compte de la présente recherche, à savoir les bovins et les ovins et caprins.

\section{Projection des effectifs d'abattage des bovins d'ici 2030}

Ainsi, selon les résultats issus de l'extrapolation, on observe d'une façon générale, le maintien des tendances à la hausse des effectifs d'abattages au niveau de chaque site d'abattage pour les espèces prises en considération dans la présente recherche. Le Tableau II présente les résultats des données par site d'abattage par rapports aux années de référence projetées.

\begin{tabular}{cccccc}
\hline \multicolumn{2}{l}{ Tableau II : Résultats de projections des effectifs d'abattage de bovins d'ici 2030 } \\
\hline \multirow{2}{*}{$\mathbf{N}^{\circ}$} & SITES D'ABATTAGE CONCERNES & \multicolumn{4}{c}{ EFFECIFS PAR ANNEE DE } \\
& & $\mathbf{2 0 1 6}$ & $\mathbf{2 0 2 0}$ & $\mathbf{2 0 2 5}$ & $\mathbf{2 0 3 0}$ \\
\hline \multirow{2}{*}{1} & L'abattoir de cotonou/ dandji cotonou & 16059 & 17632 & 19598 & 21564 \\
2 & Aire d'abattage de Houéyogbé Gbedji /Porto-Novo & 1227 & 1159 & 1074 & 989 \\
3 & Aire d'abattage de Sèmè abadame/ab-calavi & 502 & 618 & 762 & 906 \\
4 & Aire d'abattage de Agori/ kpota/ Abomey-Calavi & 669 & 822 & 1013 & 1204 \\
5 & Aire d'abattage de Houalacomey/ Abomey-Calavi & 167 & 206 & 254 & 302 \\
6 & Aire d'abattage d'Agbanou /Ouidah & 98 & 101 & 106 & 111 \\
7 & Aire d'abattage de Gomey /Ouidah & 86 & 86 & 85 & 84 \\
8 & Aire d'abattage de Zomaï/ Ouidah & 114 & 122 & 132 & 142 \\
& TOTAUX & $\mathbf{1 8 9 2 4}$ & $\mathbf{2 0 6 2 4}$ & $\mathbf{2 2 8 9 2}$ & $\mathbf{2 5 1 6 0}$ \\
\hline
\end{tabular}

Source : Résultats d'analyse, 2017

De l'analyse des données de progression, il est remarqué que la tendance d'évolution des effectifs d'abattage des bovins est à la hausse dans l'ensemble des sites d'abattage pourquoi ?, sauf sur le site d'abattage de PortoNovo, il a été observé une baisse des effectifs en 2030 qui est 989 têtes par rapport à 2011 où il avait été enregistré un effectif de 1329 têtes de bovins abattus. Les totaux de l'ensemble des sites sont globalement en hausse avec 18924 têtes de bovins en 2016, 18357 en 2015, et concernant les projections, il est attendu 20624 en 2020, 22892 en 2025 et 25160 en 2030. Ces résultats indiquent clairement qu'on peut espérer la hausse des effectifs d'abattage d'ici 2030 pour desservir les communautés du Grand Nokoué en matière de viande bovine. 


\section{Projection des effectifs d'abattage des ovins et caprins d'ici 2030}

Comme il l'a été démontré pour le cas des bovins, la projection des effectifs d'abattage des ovin et caprins a été faite, et on observe cette même tendance que pour ce qui concerne les bovins ainsi que l'indique le tableau III.

Tableau III : Résultats de projections des effectifs d'abattage de ovins et caprins d'ici 2030

Tableau III : Résultats de projections des effectifs d'abattage de ovins et caprins d'ici 2030

\begin{tabular}{clcccc}
\hline \multirow{2}{*}{$\begin{array}{c}\text { N } \\
\circ\end{array}$} & \multicolumn{2}{c}{ SITES D'ABATTAGE CONCERNES } & \multicolumn{3}{c}{ EFFECTIFS PAR ANNEE DE } \\
& \multicolumn{2}{c}{ REFERENCE } \\
\hline 1 & L'abattoir de cotonou/ dandji cotonou & $\mathbf{2 0 1 6}$ & $\mathbf{2 0 2 0}$ & $\mathbf{2 0 2 5}$ & $\mathbf{2 0 3 0}$ \\
2 & Aire d'abattage de Sèmè-Kpodji & 76401 & 107886 & 134742 & 161598 \\
3 & Aire d'abattage de Houéyogbé Gbedji /Porto-Novo & 7149 & 9184 & 11727 & 14270 \\
4 & Aire d'abattage de Agori/ kpota/ Abomey-Calavi & 4065 & 8564 & 10465 & 12366 \\
5 & Aire d'abattage d'Agbanou /Ouidah & 373 & 394 & 4290 & 4415 \\
6 & Aire d'abattage de Gomey /Ouidah & 368 & 371 & 374 & 377 \\
7 & Aire d'abattage de Zomai// Ouidah & 373 & 394 & 420 & 446 \\
\multicolumn{2}{r}{ TOTAUX } & $\mathbf{1 0 7 7 9 0}$ & $\mathbf{1 3 2 9 7 8}$ & $\mathbf{1 6 4 4 6 3}$ & $\mathbf{1 9 5 9 4 8}$ \\
\hline
\end{tabular}

Source : Résultats d'analyse, 2017

Il ressort de l'analyse des données du tableau III que la tendance à l'évolution des effectifs d'abattage des ovins et caprins est à la hausse dans l'ensemble des sites d'abattage. Les totaux de l'ensemble des sites sont globalement en hausse avec 107790 têtes de ovins et caprins en 2016, 132978 en 2020,164463 en 2025 et 195948 en 2030 selon les projections. Ces résultats indiquent clairement qu'on peut espérer la hausse des effectifs d'abattage d'ici 2030 pour desservir les communautés du Grand Nokoué en matière de viande d'ovins et caprins. En conséquence, cette évolution des effectifs d'abattage implique une augmentation des déchets qui devraient en être issus.

\section{Evaluation des quantités des contenus des panses de 2011 à 2015}

A l'issu des opérations de pesés des déchets (contenus de panse) de chaque espèce au bout de 50 jours, à raison d'une espèce de bovin et une espèce de d'ovin ou caprin chaque jour, les données ont été normalisées et consignées dans un tableau Excel comme base de données. Les différentes mesures du poids de déchets obtenues par individu et par espèce ainsi leurs poids vifs sont indiqués dans le tableau IV. 
Tableau IV : Résultats des mesures des contenus de panse de l'échantillon d'espèces et leur poids vifs

\begin{tabular}{llc|cc}
\hline $\mathbf{N}^{\circ}$ & $\begin{array}{c}\text { Poids vif des } \\
\text { bovins (Kg) }\end{array}$ & $\begin{array}{c}\text { Contenu de } \\
\text { panse de } \\
\text { bovin (Kg) }\end{array}$ & $\begin{array}{c}\text { Poids vif des } \\
\text { ovins/caprins (Kg) }\end{array}$ & $\begin{array}{c}\text { Contenu de } \\
\text { panse d'ovins } \\
\text { /caprins (Kg) }\end{array}$ \\
\hline 1 & 373,2 & 45 & 37 & 2,00 \\
2 & 375,1 & 47 & 54 & 3,5 \\
3 & 387 & 61 & 58 & 4,95 \\
- & - & - & - & - \\
- & - & - & - & - \\
49 & 369 & 42,4 & 59 & 5,55 \\
50 & 297 & 31 & 53 & 4,65 \\
\hline
\end{tabular}

Source : Travaux de terrains, 2017 ; NB : les individus et les variables sont de 1 à 50 pour chaque catégorie d'espèce

De l'analyse des données du tableau IV, on s'aperçoit du fait que les poids des contenus de panse ont varié entre 30,06 et $64,2 \mathrm{Kg}$ pour les bovins et de 2 à $6,35 \mathrm{Kg}$ pour les ovins. Les poids vifs des ont aussi varié entre 256 et $418 \mathrm{Kg}$ pour les bovins et 25 à $62 \mathrm{Kg}$ pour les ovins et caprins. Ces données ont étant insérées dans un modèle, un nuage de point a été réalisé avec les données obtenues pour chaque espèce animale. Une courbe de tendance a été ajoutée au nuage de point de chaque espèce pour permettre d'estimer le poids des déchets en fonction du poids des animaux. Le coefficient de corrélation $\mathrm{R}^{2}$ a permis de choisir la fonction qui ajuste le mieux ces données (figure 2 et 3 ).

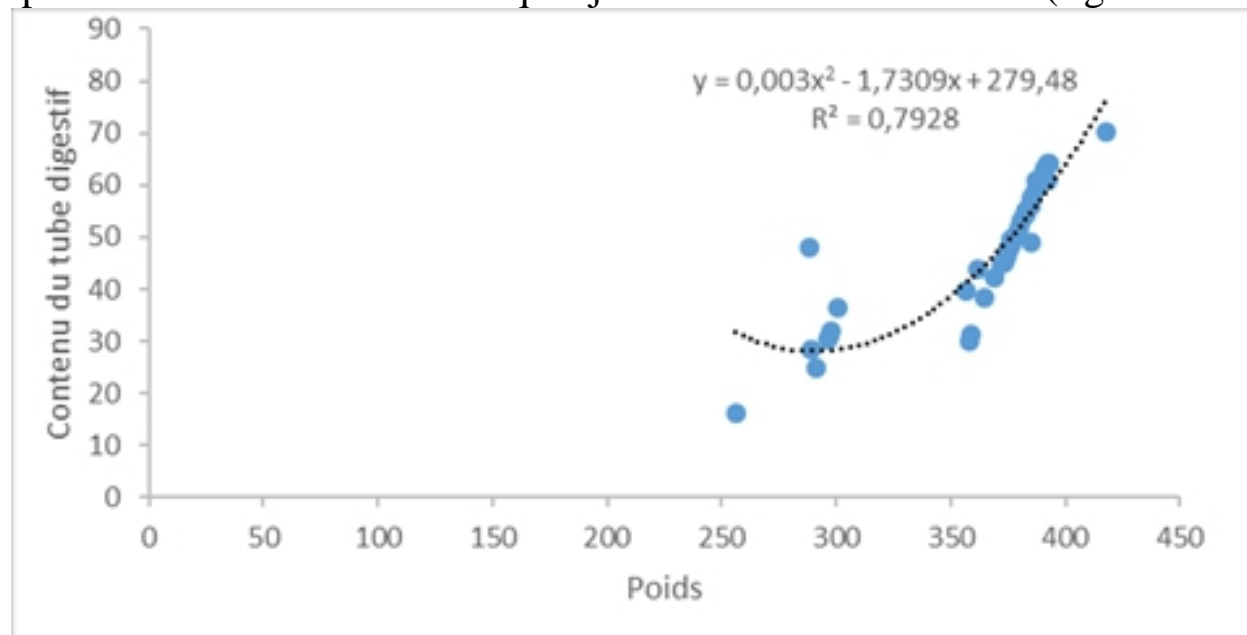

Figure 2 : Courbe de tendance et au nuage de point du lien entre le poids des déchets et le poids vif des bovins. 


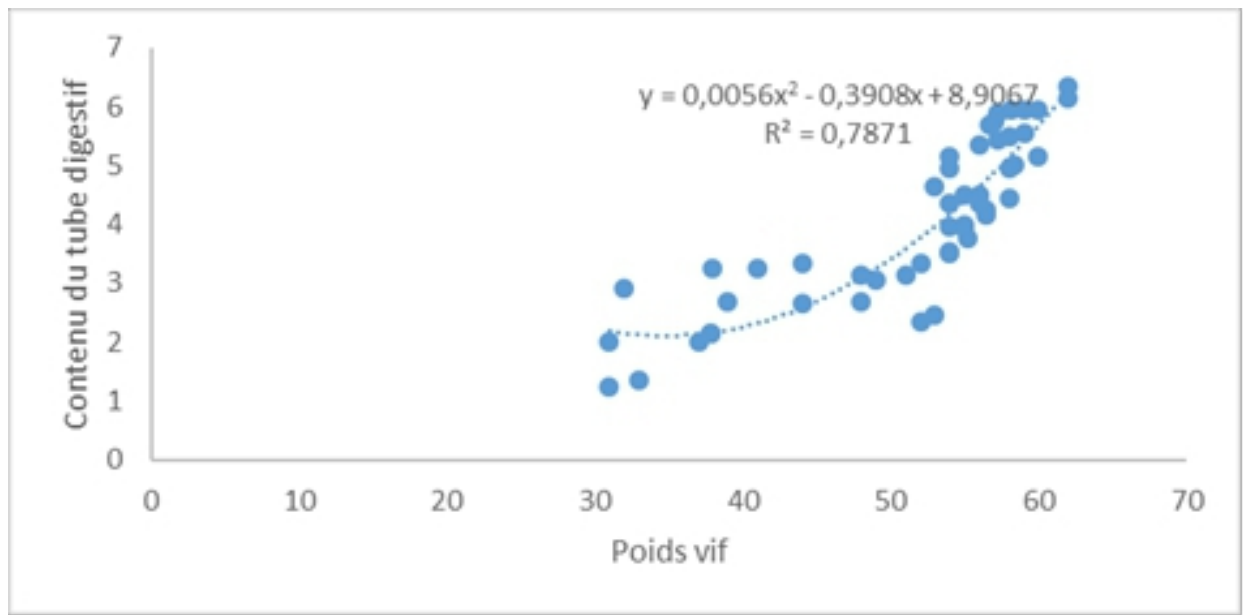

Figure 3: Courbe de tendance et au nuage de point du lien entre le poids des déchets et le poids vif des ovins et caprins.

Sources : Résultats d'analyse, 2017

Les figures 2 et 3 permettent d'estimer les valeurs des poids des déchets des bovins et des ovins et caprins en lien avec leurs poids respectifs. De l'analyse de ces figures 32 et 33, il à retenir que les $\mathrm{R}^{2}$ obtenus aussi bien pour les bovins $\left(R^{2}=0,7928\right)$, que pour les ovins et caprins $\left(R^{2}=0,7871\right)$ sont très proches de 1 . Ce qui veut dire qu'il a un lien fort et positif entre le poids de déchets et le poids vif de l'animal. L'analyse de régression effectuée montre que le poids de l'animal est fortement corrélé avec le poids des déchets recueillis après son abattage. Il est donc établi qu'il y a un lien de cause à effet entre le poids vif de l'animal avec le poids des contenus de panse. Plus le poids vif de l'animal est élevé, plus, le déchet qui en sort à l'abattage est important. Une bonne majorité des espèces animales abattues dans le Grand Nokoué sont des Zébus (Bos indicus), taurins (Bos taurus) pour la plupart venues du Nord et du centre du Bénin ainsi que les pays de l'intherland tels que le Niger et le Burkina-Faso et aussi du Nigéria. Donc, elles ont presque les conformations identiques. Cependant, en tenant en compte, d'une part, des variabilités génotypiques et phénotypiques respectivement liées aux races de bovins, ovins et caprins ainsi que leurs stades de développement pouvant influer sur la taille et le poids de l'animal et, par conséquent, sur la quantité de déchets que celuici produit à l'abattage, et d'autre part, selon la cohérence de l'hypothèse pessimiste adoptée dès le début de cet exercice, la moyenne a été retenue.Sur cette base, les poids des déchets pesés par rapport aux échantillons des 50 Bovins et 50 ovins et caprins ont été additionnés selon par catégorie et leurs moyennes déterminées en les divisant par 50. 
- Soit $\mathrm{P}_{\mathrm{bm}}$ le poids moyen de déchets de bovin en Kg On a : $\mathrm{P}_{\mathrm{bm}}=\frac{P_{b}}{50}$ (1)

Avec $\mathrm{Pb}=$ Poids total des déchets obtenus, et 50 représente le total de têtes de bovins choisies comme l'échantillon. $\mathrm{P}_{\mathrm{bm}}=\frac{2428,06}{50}=48,5612 \mathrm{~kg}$

Poids moyen de contenus de panse de bovins $=48,56 \mathrm{~kg}$ soit 0,048 tonne

- Soit $\mathrm{P}_{\mathrm{om}}$ le poids moyen de déchets d'ovin/ caprin, $\mathrm{P}_{\mathrm{om}}=\frac{P_{O}}{50}$

Avec $\mathrm{P}_{0}=$ Poids total des déchets obtenus, et 50 représente le total de têtes de ovins et caprins choisies comme l'échantillon. $\mathrm{P}_{\mathrm{bm}}=\frac{202}{50}=4,0364 \mathrm{~kg}$

Afin de déterminer la masse de déchet par année pour chaque espèce au niveau de chaque unité d'abattage, le poids moyen des déchets par espèce a été

Poids moyen de contenus de panse d'ovin/caprin $(\mathrm{Pbm})=\mathbf{4 , 0 3 6 4} \mathrm{kg}$ soit $\mathbf{0 , 0 0 4}$ tonne

multiplié par l'effectif d'espèces abattues pour l'année considérée. Cela se traduit par les formules suivantes :

- Masse annuelle de déchets de bovins = effectif annuel d'abattage de bovins $\times \mathrm{P}_{\mathrm{bm}}$ pour chaque unité d'abattage.

- Masse annuelle de déchets d'ovins/caprins = effectif annuel d'abattage d'ovins/caprins $\times \mathrm{P}_{\text {Om }}$ pour chaque unité d'abattage

Étant donné que les contenus aussi bien des bovins que des ovins et caprin sont presque de même nature, donc, ils sont destinés à faire l'objet de traitement unifié en raison de leur composition physico-chimique à peu près identiques. Ainsi, la masse de contenus de panse bovine additionnée à celle des caprins et ovins pour avoir la masse totale des déchets toutes espèces confondues par an et par unité d'abattage. Pour les unités où il n'y a qu'une catégorie d'espèce abattue, le problème ne pose pas. Mais, pour des raison d'efficacité dans la manipulation des données, les communes de Ouidah et d'Abomey-Calavi qui ont chacune trois aires d'abattage, il a été retenu d'associer les masses des unités d'abattage, afin d'avoir une masse unique annuelle déterminant le flux de déchets au niveau communal qui soit en harmonie avec les autres communes où, il n'y a qu'une seule unité d'abattage pour chacune par rapport à la période de référence (2011 à 2015) et les projections faites jusqu'en 2030. Les figures 4, 5, 6 et 7 montrent la tendance d'évolution des flux de déchets de 2011 à 2015 par commune du Grand Nokoué. 


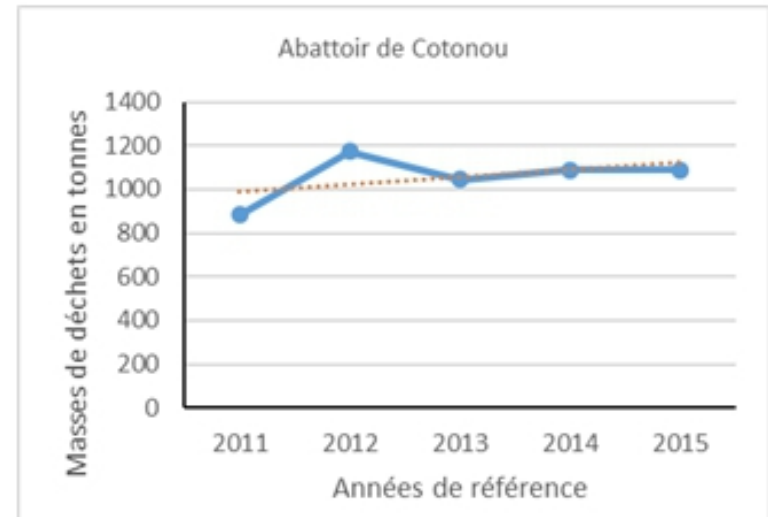

Figure 4 : Évolution des flux de déchets de 2011 à 2015 dans la ville de Cotonou

Source : Résultats d'analyse, 2017

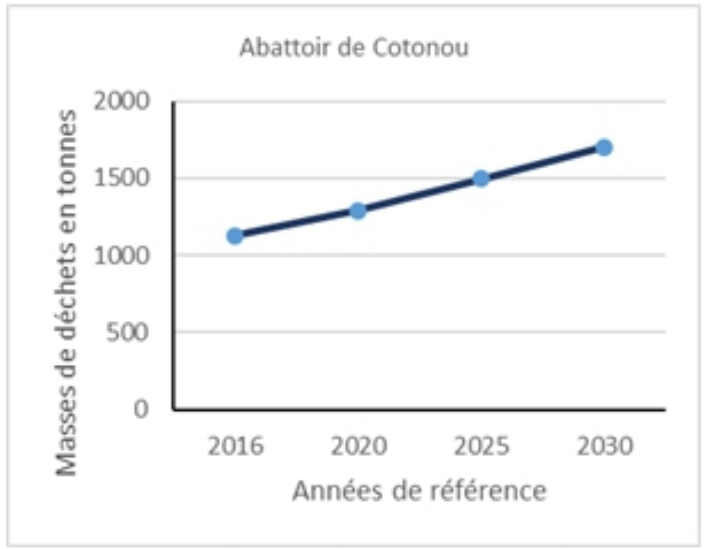

Figure 5 : Projection des flux de déchets de 2016 à 2030 dans la ville de Cotonou

Il ressort de la figure 4 que dans l'agglomération de Cotonou, l'évolution du flux annuel de la fraction organique animale cible montre une masse de déchets importante, évoluant de façon progressive et presqu'en dents de scie, allant de 883,95 tonnes en 2011, passe à 1171,60 tonnes en 2012, descend à 1045,95 en 2013 et repart à 1089,77 2014, avant de stagner à 1087,82 tonnes en 2015. En observant la courbe de tendance, d'une manière générale, on note une hausse tendancielle de la masse de déchets organique de l'agglomération de Cotonou. Les prévisions à l'horizon 2030 indiquent que la masse de ces déchets pourrait atteindre 1699,44 tonnes (figure 5).

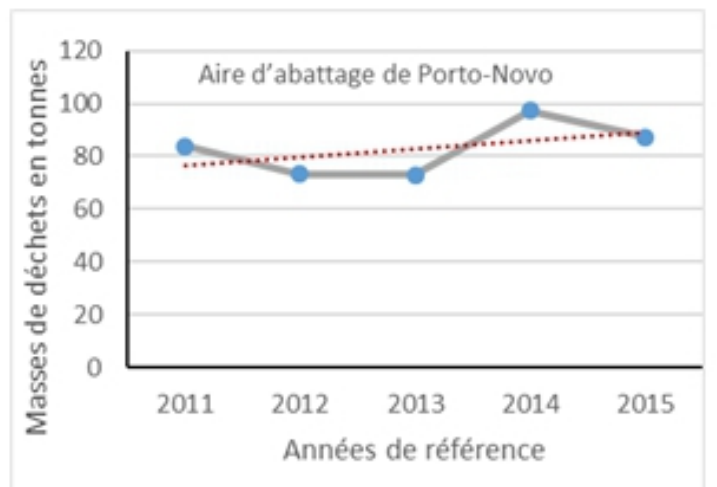

Figure 6 : Évolution des flux de déchets de 2011 à 2015 dans l'agglomération de Porto-Novo Source : Résultats d'analyse, 2017

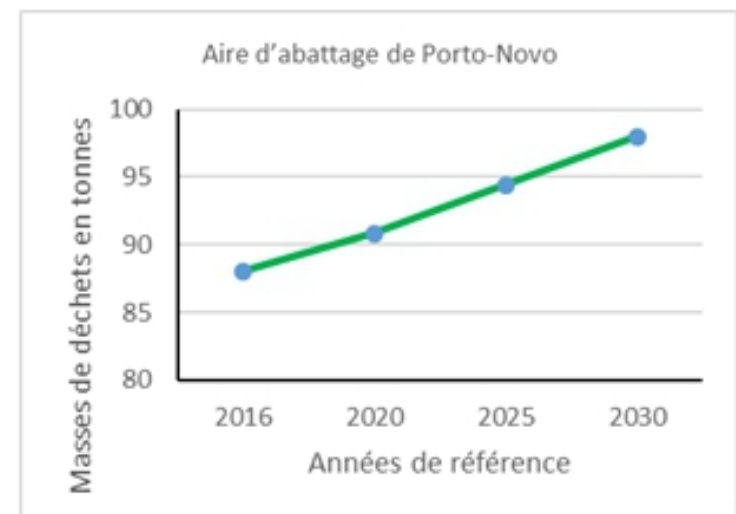

Figure 7: Projection des flux de déchets de 2016 à 2030 dans 1'agglomération de Porto-Novo

Il ressort de la figure 6 que dans l'agglomération de Porto-Novo, l'évolution du flux annuel de la fraction organique animale cible montre une masse de déchets relativement élevée, évoluant presqu'en dents de scie, allant de 83,75 tonnes en 2011, elle baisse à 72,13 tonnes en 2012, reste presque 
stable à 72,99 en 2013, avant de remonter à 97,26 tonnes 2014 puis redescend légèrement à $87,30,2015$. En observant la courbe de tendance, d'une manière générale, une hausse tendancielle légère de la masse de déchets organique dans la commune de Porto-Novo. Les prévisions à l'horizon 2030 indiquent un relatif regain de hausse qui avoisinerait les chiffres de 2014 97,94 tonnes (figure 7).

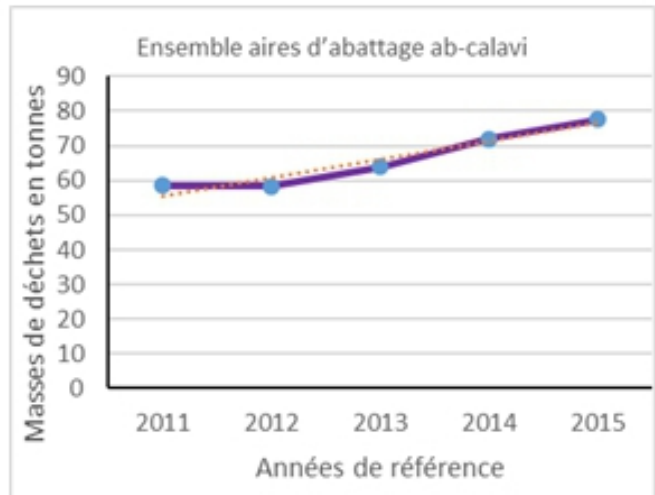

Figure 8 : Évolution des flux de déchets de 2011 à 2015 dans l'agglomération d'Abomey-Calavi Source : Résultats d'analyse, 2017

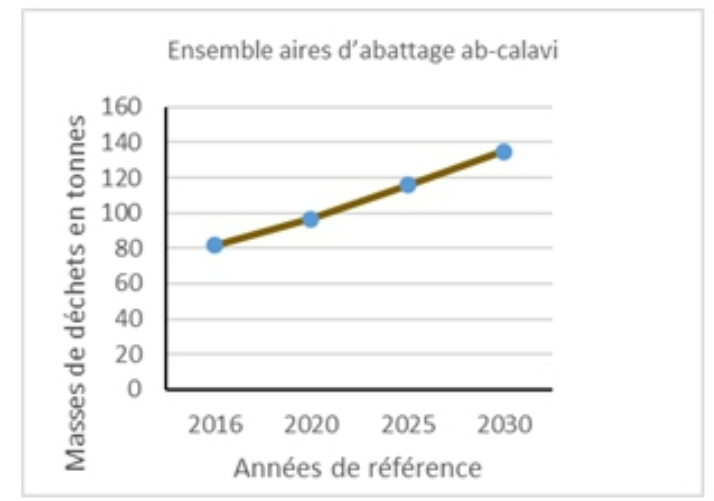

Figure 9 : Projection des flux de déchets de 2016 à 2030 dans l'agglomération d'Abomey-Calavi

Il ressort de la figure 8 que dans la commune d'Abomey-Calavi, l'évolution du flux annuel de la fraction organique animale cible montre une masse de déchets relativement élevée, évoluant de façon progressive en dents de scie, allant de 16,54 tonnes en 2011, se double presque à 30,23 tonnes en 2012, descend en continu respectivement à 25,21 et 17,54 tonnes en 2013 et 2014, avant de remonter à 26,80 tonnes en 2015. En observant la courbe de tendance, d'une manière générale, une hausse tendancielle de la masse de déchets organique dans la commune de Sèmè-Kpodji. Les prévisions à l'horizon 2030 indiquent que la masse de ces déchets pourrait atteindre 57,59 tonnes (figure 9). 


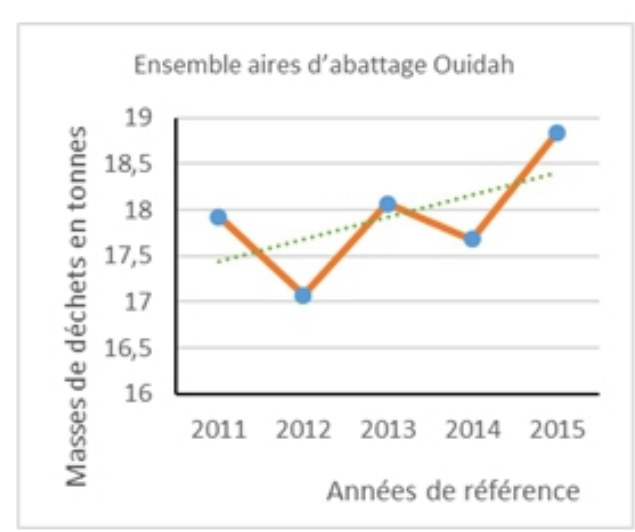

Figure 10 : Évolution des flux de déchets de 2011 à 2015 dans l'agglomération de Ouidah Source : Résultats d'analyse, 2017

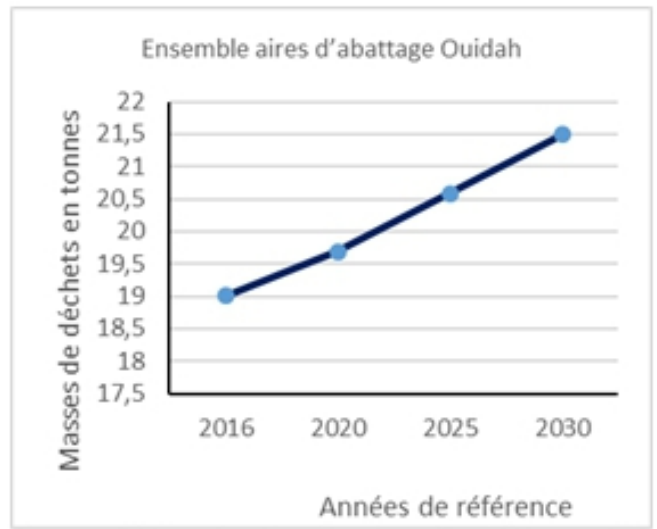

Figure 11: Projection des flux de déchets de 2016 à 2030 dans l'agglomération de Ouidah

Partant des données de la figure 10, l'analyse du flux annuel de la fraction organique animale cible dans la commune de Ouidah montre une masse de déchets relativement faible qui évolue en dents de scie, allant de 17,92 tonnes en 2011 , revient à 17,06 tonnes en 2012 , remonte à 18,06 en 2018, et se retrouve finalement à 18,83 tonnes après une légère baisse en 2014. De manière générale, la courbe de tendance indique une hausse tendancielle de la masse de déchets organique dans la commune de Ouidah. A l'horizon 2030, les projections prévoient une masse de ces déchets qui atteindrait 21,48 tonnes (figure 11).

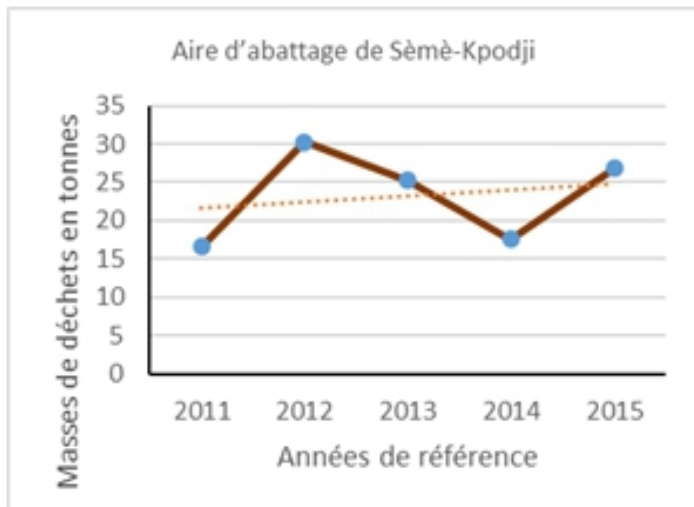

Figure 12 : Évolution des flux de déchets de 2011 à 2015 dans l'agglomération de Sèmè-Kpodji

Source : Résultats d'analyse, 2017

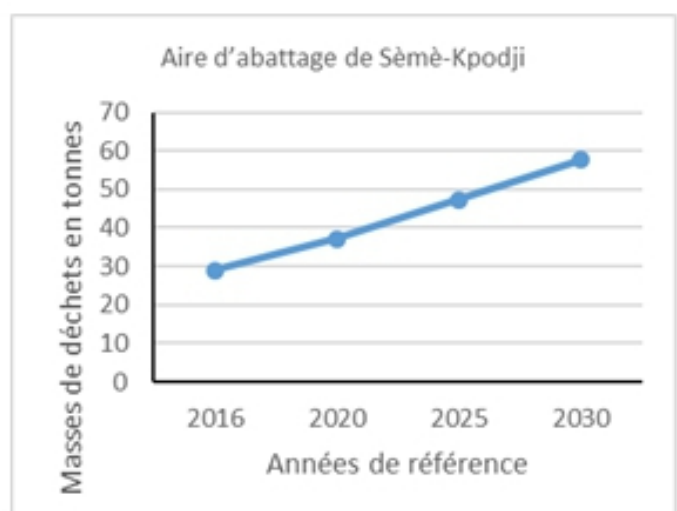

Figure 13 : Projection des flux de déchets de 2016 à 2030 dans l'agglomération de SèmèKpodji

A partir des données de la figure 12, dans la commune de SèmèKpodji, l'analyse du flux annuel de la fraction organique animale cible montre une masse de dechets relativement faible qui évolue en dents de scie, allant de 
16,54 tonnes en 2011 , se double presque à 30,23 tonnes en 2012, descend en continu respectivement à 25,21 et 17,54 tonnes en 2013 et 2014, avant de remonter à 26,80 tonnes après 2015 . En observant la courbe de tendance, d'une manière générale, une hausse tendantielle de la masse de déchets organique dans la commune de de Sèmè-Kpodji. Les prévisions à l'horizon 2030 indiquent que la masse de ces déchets pourrait atteindre 57,59 tonnes (figure 13).

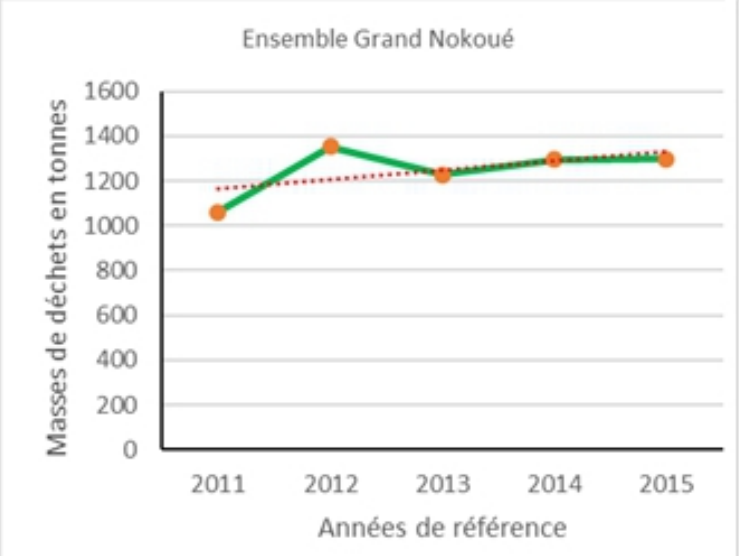

Figure 14 : Évolution des flux de déchets de 2011 à 2015 dans l'agglomération du Grand Nokoué Source : Résultats d'analyse, 2017

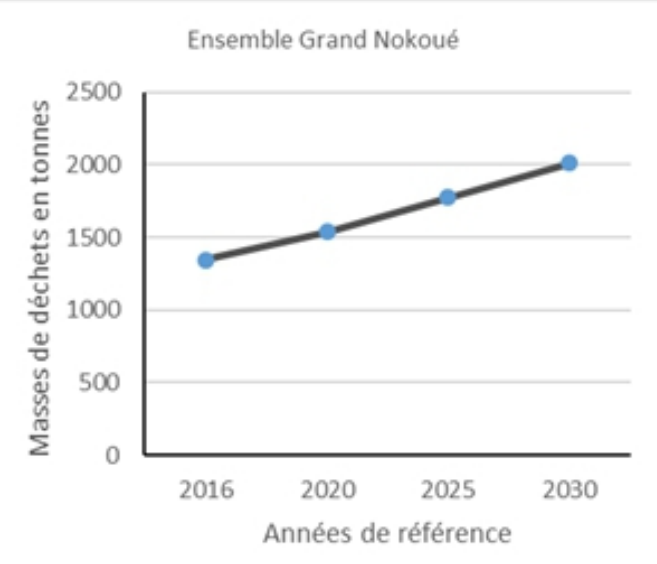

Figure 15 : Projection des flux de déchets de 2016 à 2030 dans l'agglomération du Grand Nokoué

Il ressort de la figure 14 que dans l'ensemble de l'agglomération du Grand Nokoué, l'évolution du flux annuel de la fraction organique animale cible montre une masse de déchets importante, évoluant de façon progressive et presqu'en dents de scie, allant de 1060,72 tonnes en 2011, passe à 1350,39 tonnes en 2012, évolue en baisse à 1226,20 en 2013 et repart à 1294,37 2014, pour stagner presque à 1098,82 tonnes en 2015. En observant la courbe de tendance, d'une manière générale, une hausse tendancielle de la masse de déchets organique de l'agglomération du Grand Nokoué. De l'analyse de la figure 15, les prévisions à l'horizon 2030 indiquent que la masse de ces déchets pourrait atteindre 2011,42 tonnes. La comparaison des masses fraiches de matières organiques animales de l'abattoir de Cotonou à celles des aires d'abattage prises en compte dans la présente thèse, a mis en évidence le caractère prépondérant d'activités d'abattage à l'abattoir de Cotonou, suivie de l'aire d'abattage de Porto-Novo. L'ensemble des aires d'abattage de Ouidah présente les plus faibles quantités par rapport aux autres unités d'abattage. 


\section{Potentiel de biométhanisation et de compostage des contenus de panse}

Dans le Grand Nokoué, le potentiel théorique de compostage et de biométhanisation des matières organiques animales a été estimé à 318,21 tonnes de compost et 132590,19 $\mathrm{Nm}^{3}$ de biogaz en 2011, à partir des gisements de matière organique de l'ordre de 1060,72 tonnes de matière fraiches. Il est respectivement de 405,11 tonnes et $836781,282 \mathrm{Nm}^{3}$ de compost et de biogaz en 2012, sur la base de 1350,39 tonnes de déchets organiques animales. En 2015, le potentiel de compostage et de biométhanisation sont respectivement de 389,52 de compost tonnes et $1115597,7 \mathrm{Nm}^{3}$ de biogaz en 2012, sur la base de 1298,4098 tonnes de déchets organiques animales. A 1'horizon 2030, le potentiel de compostage et de biométhanisation seraient respectivement de 603,42 tonnes de compost et $1884923,43 \mathrm{Nm}^{3}$ de biogaz, sur la base de 2011,42 tonnes de déchets organiques animales pour tout le Grand Nokoué.

Comme il l'a été souligné ci-dessus, les valeurs sont purement théoriques, elles pourraient variées en fonction des conditions environnementales, des intrants, des circuits de gisements des matières organiques, aux technologies et procédés mis en œuvre dans la digestion aérobie et anaérobie, le degré de préparation des matières premières, le niveau de contrôle et de gestion de l'exploitant. En effet, en fonction de la combinaison de ces paramètres, même dans le cas où ces déchets organiques sont nominalement équivalents, les potentiels énergétiques et le potentiel de matières fertilisantes seraient inférieures, supérieures ou égales aux valeurs théoriques énoncées. Néanmoins, il est important de remarquer que ces chiffres ont mis en perspective des opportunités d'accès aux matières fertilisantes et à l'énergie biologies à partir des gisements de contenus de panses issus des activités d'abattage dans le Grand Nokoué, principalement au profit du développement durable.

\section{Discussions}

Il ressort de cette recherche que chaque bovin abattu génère 48,5612 $\mathrm{kg}$ de déchets (contenus digestif). Il convient de rappeler que la majorité des bovins pris comme échantillon est de la race zébu et taurin. Quant aux ovins et caprins, chaque animal génère une quantité de d'environ 4,0364. kg de déchets. Ces chiffres sont proches de ceux trouvés par Hounkanri (2015) qui estime 40,5 kg pour le bovin, $3 \mathrm{~kg}$ pour les ovin/caprins. Pour Diallo (1999) et Agoma, ils sont de $30 \mathrm{~kg}$ pour le bovin et $3,5 \mathrm{~kg}$ pour les ovins/caprins. Selon Lègbagah (2009), ces valeurs de masses des de déchets représentent environ $10 \%$ du poids vif pour chaque espèce. Coulombj (1976) a estimé à $38 \mathrm{Kg}$ le poids du contenu digestif de la race bovine N'Dama. La race bovine N'Dama est une race de petit format d'environ $330 \mathrm{~kg}$ pour les mâles et $285 \mathrm{~kg}$ pour les femelles, les meilleurs taureaux ne dépassant que de peu les $450 \mathrm{~kg}$ à 
l'âge de 5 ans. Au total, ces différences observées par rapport aux poids moyens indiqués par les différents auteurs peuvent s'expliquer par le choix des approches utilisées pour la pesée des contenus digestifs ainsi que les paramètres pris en compte par ces approches, y compris les espèces animales considérées. Avec cette moyenne de 48,5612 kg de contenus de panse bovine, et $4,0364 \mathrm{~kg}$ pour les ovins et caprins, le gisement de de contenus de digestifs a été estimé à 1060 tonnes pour l'année 2011, et à 1298 tonnes pour l'année 2015 à l'échelle du Grand Nokoué. Si cette tendance est maintenue, ce gisement pourrait avoisiner 2011 tonnes d'ici 2030.

\section{Conclusion}

$\mathrm{Au}$ terme de cette recherche, on retient que les déchets organiques animaux d'abattage au sens strict sont produits dans les unités d'abattage du Grand Nokoué. Une connaissance de ces matières organiques en termes quantitatifs, constitue une étape majeure dans l'atténuation des impacts environnementaux et sanitaires, dans la contribution à l'évaluation des unités de réduction d'émission certifiées (UREC) de gaz à effet de serre (GES) par rapport aux conditions actuelles de traitement des déchets organiques, considérées comme scénario de base ou «business as usual » (BAU), et dans la planification de la logistique et de l'économie de la gestion. L'évaluation des gisements des fractions valorisables participe également des stratégies d'autofinancement et de pérennisation des actions de traitement et de valorisation dans le cadre d'une filière. Les résultats d'évaluation indiquent que les gisements des déchets organiques animaux disponibles représentent des quantités importantes sans cesse croissantes passant de 1060 tonnes pour l'année 2011 à 1298 tonnes pour l'année 2015 à l'échelle du Grand Nokoué. Si cette tendance est maintenue, cette gisement pourrait avoisiner 2011 tonnes d'ici 2030; avec fort potentiel biodégradable, donc très favorable à la méthanisation et au compostage. Cette recherche rend compte de la disponibilité des déchets organiques animaux d'abattage très adaptés à la méthanisation et le compostage en particulier. Cette recherche offre également l'opportunité aux acteurs du secteur d'avoir des données qui permettent d'envisager la mise en place d'une filière. Il s'agit de valoriser ces déchets et résidus comme source d'énergie secondaire pour des usages domestiques (cuisson, éclairage, électricité) et/ou comme sources de matières fertilisantes pour l'agriculture, notamment au profit de l'amélioration des conditions d'existence des communautés à la base. Cette recherche vient combler un vide en matière de connaissance des flux de déchets qui circule et de voir des pertes de ressources encourues annuellement par dans le secteur de cette recherche.

A l'avenir, il serait intéressant d'explorer les technologies de traitement et de valorisation de cet de type matières organiques par 
méthanisation et compostage en vue mesurer leur potentiel dans le contexte du Grand Nokoué.

\section{References:}

1. Citeretse L. (2008) : Les déchets ménagers solides de la ville de Bujumbura (Burundi): Quelles perspectives pour une gestion durable ? Master en Sciences et Gestion de l'Environnement, IGEAT, Université Libre de Bruxelles, 78 p.

2. Coulombj. (1976): La race N'Dama: quelques caractéristiques zootechniques Revue. Elev. Méd. vét. Pays trop. 29 (4) : 367-380.

3. Kapepula K. D., 1996, Composition et caractéristiques des déchets solides ménagers dans neuf villes africaines in Cahier technique : la problématique des déchets solides ménagers dans les villes africaines d'importance moyenne. Séminaire, Gembloux, Belgique, p. 96-110.

4. Lacour J (2012) : Valorisation de résidus agricoles et autres déchets organiques par digestion anaérobie en Haïti, Thèse de Doctorat de l'INSA de Lyon, Université Quisqueya, 218 p.

5. Lampila M. (1963): Experiments with alcali straw and urea. . Annales Agriculture Fenniae, vol. 2, pp.105-108.

6. Ngambi J R. (2015) : Déchets solides ménagers de la ville de Yaoundé (Cameroun): de la gestion linéaire vers une économie circulaire. Géographie. Université du Maine, 486 p

7. Onibokun Adepoju G. (2001) : La gestion des déchets urbains. Des solutions pour l'Afrique. CIRDI, Karthala, 256 p.

8. Owen E. et Jayasuriya M. C. N. (1989): Use of crop residues as animal feeds in developing countries. Research and Development in Agriculture, vol. 6, n 3, pp. 129-138.

9. Tanawa E., 1997, Une démarche pour la maîtrise de la gestion des déchets solides au Cameroun. Revue Sciences et Technologies N5, Yaoundé, $34 \mathrm{p}$.

10. Tchobanoglous G., Theisen H., Vigil S. (1993): Integrated Solid Waste Management, Engineering Principles and Management Issues. New York: McGraw Hill.

11. Wilson R. K., Pigdon W. J. (1964) : Effects of a sodium hydroxide treatment on the utilisation of wheat straw and poplar wood by rumen microorganisms. Canadian Journal of Animal Science, vol. 44, pp. 117-132. 